\title{
Preparation and Investigation of Gastro-Retentive Mucoadhesive Microspheres of Clarithromycin-Resin Complex
}

\author{
Johnson Stephin, Koland Marina* \\ NITTE (Deemed to be University), NGSM Institute of Pharmaceutical Sciences (NGSMIPS), Department of Pharmaceutics, Deralakatte, Mangalore, Karnataka, INDIA.
}

\begin{abstract}
Introduction: Helicobacter pylori infection is strongly associated with chronic gastritis and duodenal ulcers which can benefit from gastroretentive delivery of clarithromycin. Objectives: The objective of this study was to investigate mucoadhesive microspheres of the antibiotic resin complex for gastro-retention, thereby increasing the local effective concentrations of clarithromycin. Methods: Clarithromycin-resin complex granules were coated with the mucoadhesive polymers, Carbopol 934 and Polycarbophil in different ratios of 1:1, 1:2, 1:3 and 1:4 by solvent evaporation method. Prepared microspheres were subjected to characterization for particle size and shape, drug content, in vitro mucoadhesion, in vitro drug release and in vivo gastric residence time in albino rats. Results and Discussion: Particle sizes of the microspheres were found to be in the range of $83 \mu \mathrm{m}-87 \mu \mathrm{m}$. Scanning electron microscopic images of the formulation showed spherical particles with almost smooth surface morphology for carbopol microspheres. Formulations with both polymers showed mucoadhesion for a period of more than $6 \mathrm{hr}$. In vitro drug release using the USP dissolution test apparatus appeared to
\end{abstract}

follow first order kinetics. Fluorescence imaging of histo-pathological slides of rats gastric and intestinal mucosa reveal that gastric residence time was found to be longer than $6 \mathrm{~h}$ for carbopol microspheres and at least $4 \mathrm{~h}$ in the case of polycarbophil microspheres. Conclusion: Carbopol coated microspheres of ion exchange resin complexes of clarithromycin for the gastro-retentive delivery of clarithromycin can be a promising alternative to the conventional oral solid dosage forms in the control of $\mathrm{H}$. pylori induced chronic gastritis or duodenal ulcers.

Key words: Clarithromycin, Fluorescence, Gastro-retentive, H. pylori, Carbopol, Mucoadhesion

\section{Correspondence}

Dr. Marina Koland

Department of Pharmaceutics, NGSM Institute of Pharmaceutical Sciences, NITTE (Deemed to be University), Deralakatte, Mangalore-575018, Karnataka, INDIA.

Phone no: +919886042035

Email: marinakol@nitte.edu.in

DOI: 10.5330/ijpi.2020.4.78

\section{INTRODUCTION}

One of the approaches used for achieving controlled and predictable drug delivery is to control the gastric residence time. Prolonging the gastric retention will help to increase the bioavailability, reduce dose frequency and provide site-specific drug delivery. Gastro retentive drug delivery systems (GRDDS) extend the drug-releasing time by remaining in the stomach and allow both spatial and time control of drug liberation. ${ }^{1}$ Oral controlled drug delivery systems have several therapeutic advantages, such as ease of administration and better patient compliance. ${ }^{2}$ But the major disadvantage associated with such products is the inability of the dosage form to remain in the GIT for a prolonged period due to the normal gastric motility and emptying. The decreased gastric residence time of conventional oral dosage form results in incomplete drug release, which leads to reduced efficacy of the administered dose.

Several methods have already been developed to prolong the residence time and to target the mucosa. Floating, swelling, expanding and bioadhesive are some of the gastro-retentive dosage forms that have seen extensive research. ${ }^{1}$ Mucoadhesive microspheres are capable of providing controlled and spatial drug delivery. They are microparticles or microcapsules which are in the size range of 1 to $1000 \mu \mathrm{m}$. They may be entirely made of a mucoadhesive polymer or can be coated with a mucoadhesive polymer. ${ }^{3}$

Ion exchange resins in microsphere form can be used to prolong gastric residence time of the incorporated drug by virtue of their mucoadhesive property. Besides having the advantages of taste masking and improving the drug stability, they can also be used in controlled drug delivery systems. ${ }^{4}$ Ion exchange resins are cross linked, water-insoluble polymers, carrying ionizable functional groups and are known to have excellent distribution in the gastric cavity. They are also high molecular weight water-insoluble polymers and are not absorbed by the body and therefore inert. ${ }^{5}$ Drug resin complexes are produced by loading the drug into the resin by an exchanging reaction and the drug is released from the resinate by exchanging with similarly charged ions in the gastrointestinal fluid. ${ }^{6}$ These systems increases the localized drug delivery due to the adhesion of ionic resins to gastric mucosa. When this ion exchange resin-drug complex is encapsulated using mucoadhesive polymers such as polycarbophil and carbapol 934, gastric residence time was further improved. For the preparation of an optimized drug-resin complex, factors such as polymer ratio, effect of drug to resin ratio and effect of $\mathrm{pH}$ on drug resin complexation would be considered.

Helicobacter pylorus (H. pylori) is the causative organism of both acute and chronic gastritis and is considered to be sensitive to broad spectrum antibiotics and certain macrolides such as clarithromycin. ${ }^{7}$ The main drawback of gastric ulcer treatment is the limited contact time with the target site when administered in conventional dosage form. ${ }^{8}$ Antibiotics that are used for the treatment of gastric ulcer disease are mostly meant to be targeted to the stomach. However, the conventional oral drug delivery systems such as tablets have disadvantages such as limited residence time in the gastrointestinal tract and also inefficient drug uptake by the gastric mucosa. Clarithromycin is the most common and single most potent macrolide antibiotic indicated in the treatment of $H$. pylori infections and is generally administered as granules or tablets. ${ }^{9}$ In order to achieve the desired anti-bacterial levels in patients, local diffusion of 
the drug in the mucosa is essential; therefore the development of oral clarithromycin dosage form with prolonged gastric residence time is important. Prolonging the gastric residence time is the primary objective of the study in order to get maximum effect of the drug in the targeted site. Thus the adherence of the microspheres to the mucosa will result in more localized drug release which helps to maintain the bacterial level to a minimum.

Clarithromycin is a free base with a pKa of 8.7 and low water solubility of about $0.342 \mu \mathrm{g} / \mathrm{mL}$. The solubility increases in acidic $\mathrm{pH}$ due to the ionization of its dimethylamino moiety, which is its only ionizable group. ${ }^{10}$ Therefore the drug would be suitable for complexation with an acid cation exchange resin at an acidic $\mathrm{pH}$. Studies show that clarithromycin undergoes rapid degradation in the acidic $\mathrm{pH}$ of the stomach to form decladinosyl clarithromycin and clarithromycin 9 , 12-hemiketal. ${ }^{11}$ Hence protection of the drug from acid degradation is crucial when formulating as a gastro-retentive dosage form so as to improve its bioavailability. One approach that can be used to protect clarithromycin from acidic degradation and provide sustained release is to incorporate the drug into mucoadhesive or bioadhesive microspheres. ${ }^{12}$ In our investigation, we have coated the drug-resin complex particles with mucoadhesive polymers such as Carbopol 934 and Polycarbophil. The use of clarithromycin as mucoadhesive microspheres of drug-resin complex will result in the sustained release of the drug locally at the site of $H$. pylori infection while protecting the drug from gastric degradation. The present work was undertaken to formulate and evaluate mucoadhesive microspheres of clarithromycin resin complex for gastric retention.

\section{MATERIALS AND METHODS}

Clarithromycin was supplied as a gift sample by Cipla Pvt. Ltd. (Goa, India). Carbopol 934 and Polycarbophil used for the mucoadhesive coating of the drug - resin complex were procured from Loba Chem. Pvt. Ltd and Yarrow Chem. Pvt. Ltd., Mumbai, respectively. The ion exchange resin, Tulsion 335 was obtained from Thermax. Pvt. Ltd. Pune. Magnesium stearate used to prevent aggregation of microspheres was procured from Yarrow Chem. Pvt. Ltd., Mumbai. All other solvents and chemicals used were of analytical grade.

\section{Formulation of mucoadhesive microspheres of clarithromycin}

\section{Purification of resin}

The weak acid cation exchange resin that we have used for our formulation is Tulsion 335 (Polacrilex). A highly purified crosslinked polyacrylic copolymer in Hydrogen form, Tulsion was first made free of interfering ions by washing with distilled water. The wet resin was activated by $300 \mathrm{ml}$ of $0.1 \mathrm{~N} \mathrm{HCl}$ and later washed with distilled water. After filtration, it was then dried in a vacuum oven at $60^{\circ} \mathrm{C}$ till the moisture content become below 5\%. It was then rinsed with methanol and then activated with $1 \mathrm{~N} \mathrm{HCl}$ again, washed with distilled water and dried in hot air oven till the moisture content became below 5\%

\section{Preparation of drug-resin complex}

Clarithromycin was mixed with tulsion-335 in the drug-resin ratio 1:1, 1:2 and 1:3. Two hundred $\mathrm{ml}$ of the distilled water was added in to the mixture and stirred for five hours with magnetic stirrer to allow complete complexation of drug with resin. Drug-resin complex so obtained was filtered by vacuum filtration and the residue was washed with distilled water. The prepared resinate was dried in hot air oven at $60^{\circ} \mathrm{C}$ till the moisture content was below $5 \%$. Unbound drug in the filtrate was estimated at $263 \mathrm{~nm}$ and the percentage drug loading was calculated.
Different ratios of drug to resin were studied to get maximum drug loading. The maximum drug bound resinate was taken as the optimum complex and used for further studies. ${ }^{13}$

\section{Optimization of $\mathrm{pH}$ of the drug-resin complex}

Drug and resin were weighed accurately and drug-resin complex was prepared according to the above procedure. The $\mathrm{pH}$ of the loading medium was adjusted to 1.0 using standard solution of $0.1 \mathrm{~N} \mathrm{HCl}$ and sodium hydroxide and stirred for $8 \mathrm{hr}$. The percentage of drug bounded was determined. Similarly, drug loading at $\mathrm{pH} \mathrm{2,4,5}$ and 6 was also determined.

\section{Mucoadhesive coating of drug - resin complex to prepare microspheres}

Mucoadhesive microspheres containing drug-resin complex were prepared by the solvent evaporation method. Carbopol 934 and Polycarbophil were used as the mucoadhesive polymers in the ratio $1: 1$, 1:2, 1:3 and 1:4 keeping the amount of drug-resin complex constant. The mucoadhesive polymer was dissolved in $15 \mathrm{ml}$ of ethanol and the drug-resin complex was added into the polymer solution. The polymer solution was mixed thoroughly and poured into $100 \mathrm{ml}$ of liquid paraffin containing magnesium stearate. Magnesium stearate helped to prevent aggregation of the particles. The above mixture was stirred using mechanical stirrer until ethanol is evaporated completely. The dispersion was filtered by vacuum filtration and microspheres were dried at $45^{\circ} \mathrm{C}$ for one hour. ${ }^{14}$

\section{Evaluation of mucoadhesive microspheres Size and shape analysis}

Size analysis was carried by Optical Microscopy using the eye piece and stage micrometer. The particle size of 150 microspheres was determined randomly and the average particle size is calculated using the following equation:

$$
\text { Average size }=\Sigma \mathrm{nd} / \Sigma \mathrm{n}
$$

Where $\mathrm{n}$ is the number of microspheres and $\mathrm{d}$ is the size of microsphere..$^{15,16}$

Scanning electron microscopy (SEM) was carried out in order to assess the shape and morphology of the sample. Uncoated microspheres of drug-resinate complexes, as well as carbopol coated and polycarbophil coated microspheres, were examined.

\section{Drug content estimation}

The content of clarithromycin in the formulation was determined by UV spectrophotometry. For this purpose, $100 \mathrm{mg}$ of the microsphere formulation was extracted using $0.1 \mathrm{~N} \mathrm{HCl}$. This dispersion was mixed using vortex stirrer for $15 \mathrm{~min}$ and after dilution with $0.1 \mathrm{~N} \mathrm{HCl}$ absorbance was measured at $263 \mathrm{~nm}$.

\section{In vitro evaluation of mucoadhesion}

Freshly excised sheep stomach mucosa of area of $6 \mathrm{~cm}^{2}$ collected within one hour of killing the animal was fixed to a glass slide using cyanoacrylate glue. ${ }^{17}$ A small quantity of microspheres was spread on the surface of the moist mucosa and the number of microspheres was counted under an optical microscope using $45 \mathrm{x}$ magnification. The glass slide with the attached mucosa was hung on the horizontal bars of the disintegration test apparatus into a one litre beaker containing $900 \mathrm{ml}$ of $0.1 \mathrm{~N} \mathrm{HCl}$. The apparatus was operated so that the glass slide moved up and down in the fluid in the beaker maintained at $37^{\circ} \mathrm{C}$ by the external bath. At suitable time intervals, the glass slide was removed and the number of microspheres remaining on the mucosa was counted 
under the microscope. The percentage mucoadhesion was calculated as percentage of microspheres remaining on the mucosa at each time interval.

\section{In vitro drug release studies}

In vitro drug release from mucoadhesive microspheres was determined using the Type I USP dissolution apparatus. Mucoahesive microspheres equivalent to $100 \mathrm{mg}$ drug was weighed and filled into a capsule and placed in the basket. The dissolution medium used was $900 \mathrm{ml}$ of $0.1 \mathrm{~N} \mathrm{HCl}$ maintained at $37 \pm 0.5^{\circ} \mathrm{C}$ and stirred at $100 \mathrm{rpm}$. Samples of the fluid was withdrawn at suitable time intervals and were replaced with equal volumes of fresh medium and each sample was analyzed spectrophotometrically at $263 \mathrm{~nm}$.

\section{In vivo gastrointestinal transit study}

The protocol for the in vivo study (Ref: NGSM/IAEC/2016-17/10), was approved by the institutional animal ethics committee (IAEC). Gastrointestinal transit study was carried out using albino rats. For the purpose of visualizing the movement of the microspheres in the GIT, the drug was replaced with sodium fluorescein.

Six rats were divided into two groups of three rats each. Rats were fasted for $24 \mathrm{~h}$ before administration of the formulation and they were allowed free access to water. These mucoadhesive microsphere formulations of fluorescein-resin complex were administered in $2 \mathrm{ml}$ of water directly to the stomach of conscious rats by a syringe fitted to a gastric cannula. ${ }^{14}$ Each group received an optimized formulation of the carbopol or polycarbophil coated microspheres. After 1, 3 and $6 \mathrm{hr}$, a rat from each group was rendered unconscious by means of ether and sacrificed by cervical dislocation. Stomach and small intestine of each animal were excised and segments of stomach, duodenum, jejunum and ileum were scanned under fluorescence microscopy to determine the presence of the microspheres. ${ }^{13}$

\section{RESULTS}

\section{Formulation of mucoadhesive microspheres of clarithromycin \\ Preparation of drug resin complex}

The drug-resin complex (Resinate) was prepared in different drug: resin ratio such as 1:1, 1:2 and 1:3. Stirring time and drug: resin ratios are the two critical factors that affect the ion exchange process. The different Drug: Resin ratios, stirring time and the observed percentage drug loading are given in Table 1 .

Table 1: Data for optimization of drug: resin ratio and stirring time for drug-resin complexation.

\begin{tabular}{cccccc} 
& & \multicolumn{4}{c}{ Percentage drug complexed at $\mathrm{T} h$ of stirring* } \\
\cline { 3 - 6 } Resin & $\begin{array}{c}\text { Drug: } \\
\text { Resin } \\
\text { ratio }\end{array}$ & $\mathrm{T}=5 \mathrm{~h}$ & $\mathrm{~T}=6 \mathrm{~h}$ & $\mathrm{~T}=7 \mathrm{~h}$ & $\mathrm{~T}=8 \mathrm{~h}$ \\
\hline \multirow{2}{*}{ Tulsion } & $1: 1$ & $71.74 \pm 0.52$ & $76.21 \pm 0.77$ & $78.32 \pm 1.32$ & $82.31 \pm 1.31$ \\
335 & $1: 3$ & $75.21 \pm 1.10$ & $78.75 \pm 1.02$ & $81.44 \pm 1.45$ & $86.17 \pm 0.92$ \\
\hline
\end{tabular}

${ }^{\star}$ Mean and S.D. of ' $n$ ' determinations, $n=3$

$\mathrm{T}=$ time in hours

\section{Optimization of $\mathrm{pH}$ of the drug-resin complex}

The $\mathrm{pH}$ of the drug solution to which the resin was added also influenced drug loading, the ionization of the resin and drug and its solubility. Since clarithromycin is a weak base with a $\mathrm{pKa}$ of 8.7 , it is mostly ionized at acidic $\mathrm{pH}$ which means greater aqueous solubility and therefore favours binding with the resin. ${ }^{18}$ Table 2 shows the composition of different formulations with drug content.

\section{Mucoadhesive coating of drug - resin complex to prepare microspheres}

The microspheres coated with mucoadhesive polymers (Carbopol and Polycarbophil) were prepared according to the procedure given in methodology. Polymers were used in different ratios, keeping the amount of drug resin complex constant. Drug: polymer ratios such as $1: 1,1: 2,1: 3,1: 4,1: 5$ and 1:6, were studied. The composition of different formulations is represented in Table 3 .

\section{Evaluation of mucoadhesive microspheres Size and shape analysis}

Optical microscopic analysis was performed to determine the average size of mucoadhesive microspheres. The average size of mucoadhesive microspheres of formulations with carbopol was found to be in the range $76.20-87.19 \mu \mathrm{m}$ and average particle size of formulations with polycarbophil was found to in the range $74.19-77.97 \mu$ mas shown in Table 3. From the results of microsphere size analysis, it was found that as the concentration of the polymer increases, particle size also increased. Shape and morphology of the microspheres were examined using SEM. The images of uncoated and coated microspheres of both carbopol and polycarbophil are given in Figure 1.

\section{Drug content estimation}

Results of drug content values are shown in Table 3. Among all the formulations F4 and F8 showed maximum entrapment efficiency.

\section{In vitro evaluation of mucoadhesion}

Overall, carbopol microspheres showed slightly better mucoadhesion as compared to those of polycarbophil, with $65-68 \%$ remaining on the mucosa at the end of $6 \mathrm{~h}$ compared to the $60-61 \%$ mucoadhesion of the latter. The trend in mucoadhesion with time for all formulations is shown in Figure 2.

\section{In vitro drug release studies}

The drug release pattern of all the formulations as can be observed from Figures 3 and 4 showed small differences from each other which may be directly linked to the polymer concentration. The maximum percentage

Table 2: Effect of drug solution pH on percentage drug complexed.

\begin{tabular}{cccc}
\hline Resin & $\begin{array}{c}\text { Drug resin ratio } \\
\text { and stirring time }\end{array}$ & $\begin{array}{c}\text { pH of the loading } \\
\text { medium }\end{array}$ & $\begin{array}{c}\text { \% Drug } \\
\text { complexed* }\end{array}$ \\
\hline & & 1 & $62.31 \pm 1.14$ \\
Tulsion 335 & $1: 2$ & 2 & $79.12 \pm 1.04$ \\
& $8 \mathrm{~h}$ & 4 & $85.63 \pm 2.10$ \\
& & 5 & $76.78 \pm 1.04$ \\
& 6 & $71.23 \pm 0.60$ \\
& & $46.58 \pm 1.07$ \\
\hline
\end{tabular}

${ }^{*}$ Mean and S.D. of ' $n$ ' determinations, $n=3$ 
drug release from F5 and F6 were $92.28 \%$ and $90.65 \%$ respectively and that of $\mathrm{F} 1$ and $\mathrm{F} 2$ were $87.25 \%$ and $81.23 \%$ respectively.

\section{Kinetic analysis of in vitro drug release studies}

The data obtained from the in vitro drug release study were fitted to different mathematical models like zero order, first order, Higuchi and Korsmeyer-Peppas. Accordingly, the drug release kinetics and release mechanisms can be predicted. ${ }^{19}$ The results of the kinetic analysis of the in vitro drug release data of all the formulations are given in Table 4.

\section{In vivo gastrointestinal transit study}

On the basis of the in vitro drug release data, formulations F1 and F5 which showed the maximum drug release at the end of $12 \mathrm{~h}$ were selected for the in vivo study. The formulations were subjected to the gastrointestinal transit studies in rats using fluorescence microscopy and the images were recorded in Figure 5.

\section{DISCUSSION}

In the preparation of the drug-resin complex, it was observed that with increase in time of stirring of the loading solution, the drug-resin complication was increased with maximum being achieved within 8 h. Also, when the concentration of ion-exchange resin was increased, the percentage of drug loading also increased. Increase in the amount of resin increases the amount of drug uptake from the solution but decreases the amount of drug per $100 \mathrm{mg}$ of the complex. Drug loading for ratios above 1:2 was not very significant and were not considered for further studies, since they required considerably larger amounts of the resin. Thus, drug: resin ratio of 1:2 was selected as the optimized one.
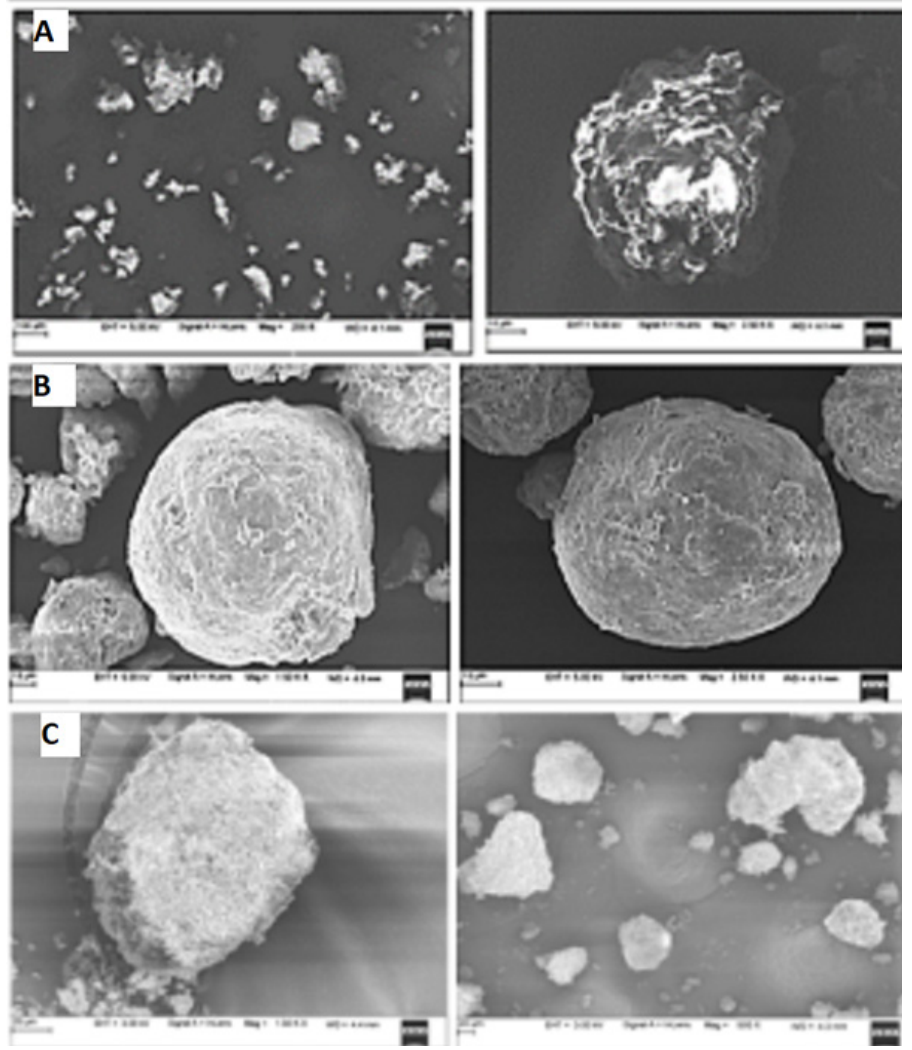

Figure 1: SEM images of uncoated microspheres of drug - resin complex (A), carbopol coated microspheres (B) and polycarbophil coated microspheres (C).

Table 3: Composition of different coated formulations with mean particle size and drug content.

\begin{tabular}{cccccccc}
\hline $\begin{array}{c}\text { SI } \\
\text { no: }\end{array}$ & $\begin{array}{c}\text { Muco- } \\
\text { adhesive } \\
\text { polymer }\end{array}$ & Formulation code & $\begin{array}{c}\text { Ratio of drug } \\
\text { resin complex: } \\
\text { polymer }\end{array}$ & $\begin{array}{c}\text { Amount of } \\
\text { drug-resin } \\
\text { complex (g) }\end{array}$ & $\begin{array}{c}\text { Amount of } \\
\text { polymer(g) }\end{array}$ & $\begin{array}{c}\text { Average particle } \\
\text { size }(\mu \mathrm{m})^{*}\end{array}$ & $\begin{array}{c}\text { Percentage drug } \\
\text { content* }\end{array}$ \\
\hline & & F1 & $1: 1$ & 0.5 & 0.5 & $76.20 \pm 1.22$ & $84.52 \pm 0.13$ \\
& & F2 & $1: 2$ & 0.5 & 1 & $84.35 \pm 3.13$ & $85.23 \pm 0.17$ \\
1 & Carbapol 934 & F3 & $1: 3$ & 0.5 & 1.5 & $86.58 \pm 2.02$ & $86.45 \pm 0.24$ \\
& & F4 & $1: 4$ & 0.5 & 2 & $87.19 \pm 1.25$ & $87.21 \pm 0.11$ \\
& & F5 & $1: 1$ & 0.5 & 0.5 & $74.19 \pm 2.42$ & $83.51 \pm 0.23$ \\
& & F6 & $1: 2$ & 0.5 & 1 & $75.99 \pm 3.35$ & $84.40 \pm 0.24$ \\
2 & Polycarbophil & F7 & $1: 3$ & 0.5 & 1.5 & $74.58 \pm 1.55$ & $84.31 \pm 0.15$ \\
& & F8 & $1: 4$ & 0.5 & 2 & $77.79 \pm 2.16$ & $85.26 \pm 0.14$ \\
\hline
\end{tabular}

${ }^{*}$ Mean and S.D. of ' $n$ ' determinations, $n=3$

Table 4: Data for release kinetics of clarithromycin from carbopol and polycarbophil microspheres.

\begin{tabular}{|c|c|c|c|c|c|}
\hline \multirow[b]{2}{*}{ Formulation } & \multicolumn{4}{|c|}{ Regression Coefficient $\left(R^{2}\right)$} & \multirow{2}{*}{$\begin{array}{c}\text { ' } n \text { ' values for } \\
\text { Korsemeyer-Peppas } \\
\text { equation }\end{array}$} \\
\hline & Zero order & First order & Higuchi model & $\begin{array}{c}\text { Korsemeyer-Peppas } \\
\text { Model }\end{array}$ & \\
\hline F1 & 0.9353 & 0.9784 & 0.9834 & 0.9623 & 0.4732 \\
\hline F2 & 0.9421 & 0.9815 & 0.9863 & 0.9773 & 0.5052 \\
\hline F3 & 0.9640 & 0.9835 & 0.9621 & 0.9373 & 0.5043 \\
\hline F4 & 0.9676 & 0.9881 & 0.9657 & 0.9368 & 0.5300 \\
\hline F5 & 0.9701 & 0.9881 & 0.9633 & 0.9582 & 0.5541 \\
\hline F6 & 0.9466 & 0.9818 & 0.9759 & 0.9643 & 0.5028 \\
\hline F7 & 0.9500 & 0.9816 & 0.9722 & 0.9581 & 0.5172 \\
\hline F8 & 0.9464 & 0.9788 & 0.9723 & 0.9556 & 0.5156 \\
\hline
\end{tabular}




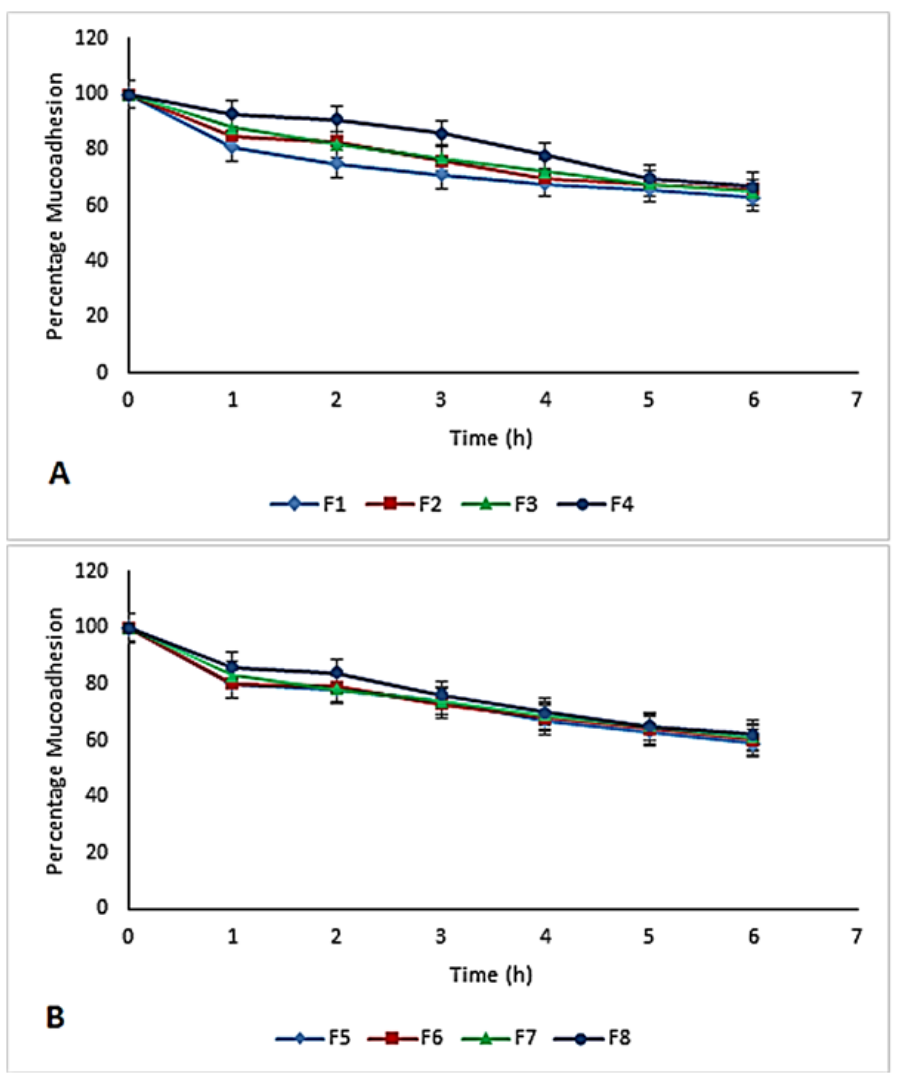

Figure 2: Trend of mucoadhesion of microspheres of formulations F1-F4 (A) and F5-F8 (B). The data represents mean \pm SD with $n=3$.

Percentage drug loading is found to be greater at lower $\mathrm{pH}$ values and decreased with increase in $\mathrm{pH}$. The percentage drug bound was found to be maximum (85.63\%) at $\mathrm{pH} 3.0$. Tulsion 335 is a cross linked polyacrylic copolymer and is a weak acid cation exchange resin with a weak acid functionality $(-\mathrm{COOH})$ which will show better ionization at lower $\mathrm{pH}$. Thus $\mathrm{pH} 3.0$ of the drug solution was considered optimum for complexing with the resin. ${ }^{20}$

It was found that as the mucoadhesive polymer concentration increases, size of the microspheres also increased. For drug-polymer ratios above 1:4, the formulation coagulated due to the large amount of polymer, compared to the amount of drug taken and irregular shaped microparticles were formed. Therefore drug-polymer ratios of 1:1, 1:2, $1: 3$ and 1:4 were selected as suitable for the preparation of mucoadhesive microspheres.

SEM images indicated that the surfaces of the coated microspheres were smooth, whereas the uncoated microparticles showed irregular surfaces. The microspheres coated with carbopol was found to be more spherical compared to that of polycarbophil. Surface of the carbopol microspheres were found to be smoother than those of polycarbophil. From the SEM images of coated and uncoated microspheres for both polymers, it is clear that the microspheres obtained spherical shape after coating with the polymers.

An increase in drug content was observed by increasing the polymer concentration in both carbopol and polycarbophil formulations. The higher drug entrapment in F4 and F8 may be because an increase in polymer concentration increased the entrapment efficiency by restricting the release of the drug by dissociation from the drug resin complex. Higher concentrations of polymer used for coating microspheres will increase the barrier effect or resistance to the diffusion of drug.

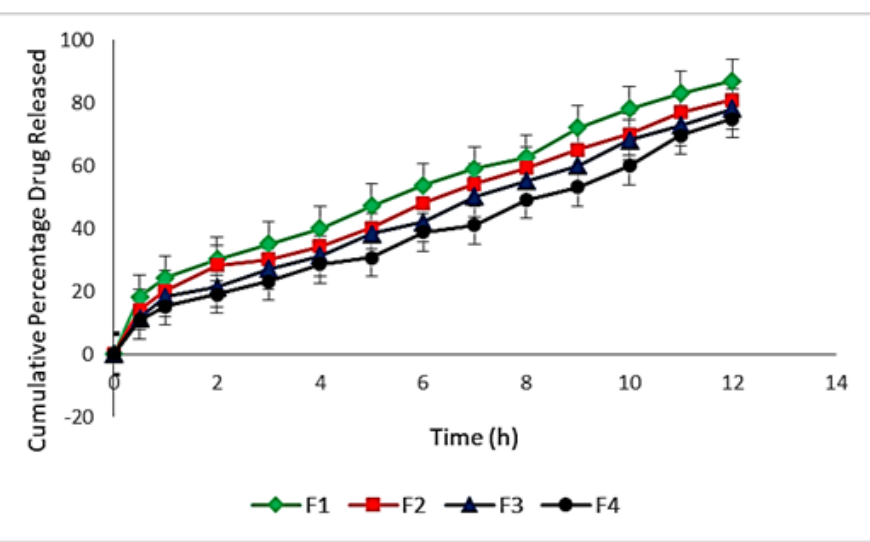

Figure 3: In vitro drug release profiles of carbopol microspheres in $0.1 \mathrm{~N} \mathrm{HCl}$. The data represents mean \pm SD with $n=3$.

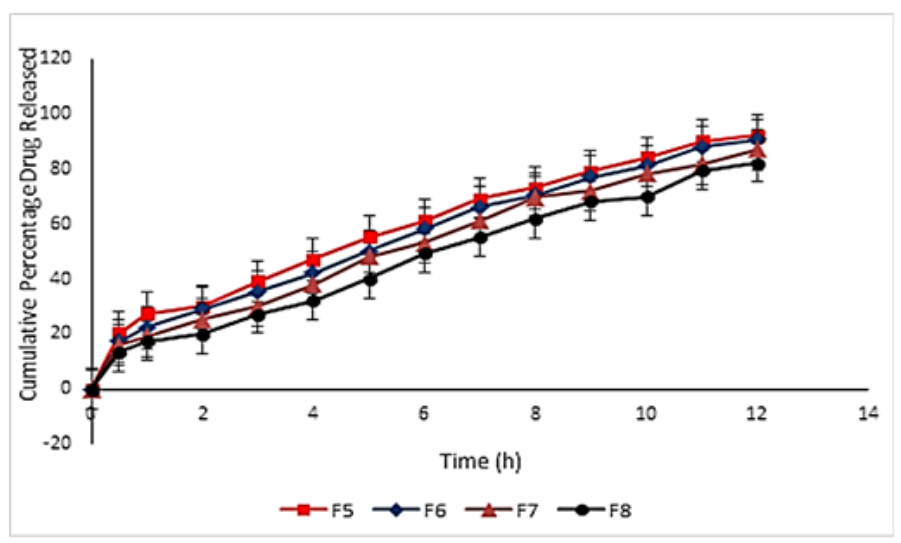

Figure 4: In vitro drug release profiles of polycarbophil microspheres in $0.1 \mathrm{~N}$ $\mathrm{HCl}$. The data represents mean \pm SD with $n=3$.

Mucoadhesion of the microspheres is important for enabling their retention on the gastric mucosal surface. Localization of the drug incorporated microspheres on the mucosa ensures adequate local concentrations of the antibiotic for the suppression of the H. pylori. ${ }^{21}$ It is observed that the retention of the microspheres on the mucosa increased with increase in the concentration of the mucoadhesive polymer. However, differences in percentage mucoadhesion between formulations of each type of polymer were small and beyond $5 \mathrm{hr}$ were not very significant. This was more apparent for carbopol microspheres and not significantly so for the polycarbophil microspheres. The superior mucoadhesion properties of carbopol could be attributed to the fact that the carboxyl groups in the polymer bind more strongly with the oligosaccharide chains of the mucin molecules in the mucus by hydrogen bonding. ${ }^{22,23}$ Mucoadhesion is also influenced by the $\mathrm{pH}$ of the medium by virtue of the effect of the latter on the degree of hydration and solubility. ${ }^{24}$ Bera K et al. has reported that carbopol 934 formulations showed greater mucoadhesion in acidic $\mathrm{pH}$ than the alkaline $\mathrm{pH}$ of the intestine. Therefore, the microspheres would have a greater potential of retention on the stomach mucosa. ${ }^{25}$

During in vitro release, the resin-drug complex on coming in contact with the release medium, provides slow diffusion of the released drug through the polymer layer in which the drug-resin complex is encapsulated. ${ }^{26}$ Generally an increase in mucoadhesive polymer concentrations can result in decreasing rates of drug release due to the resistance to the diffusion of drug offered by the gelled surface coating of the microspheres. This decreases the effective diffusion coefficient and 


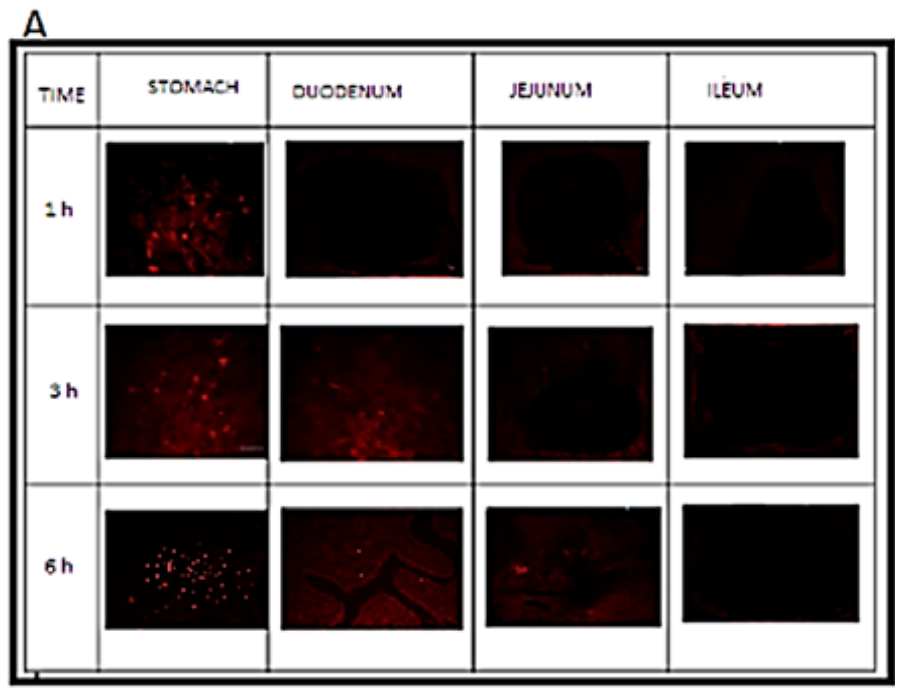

B

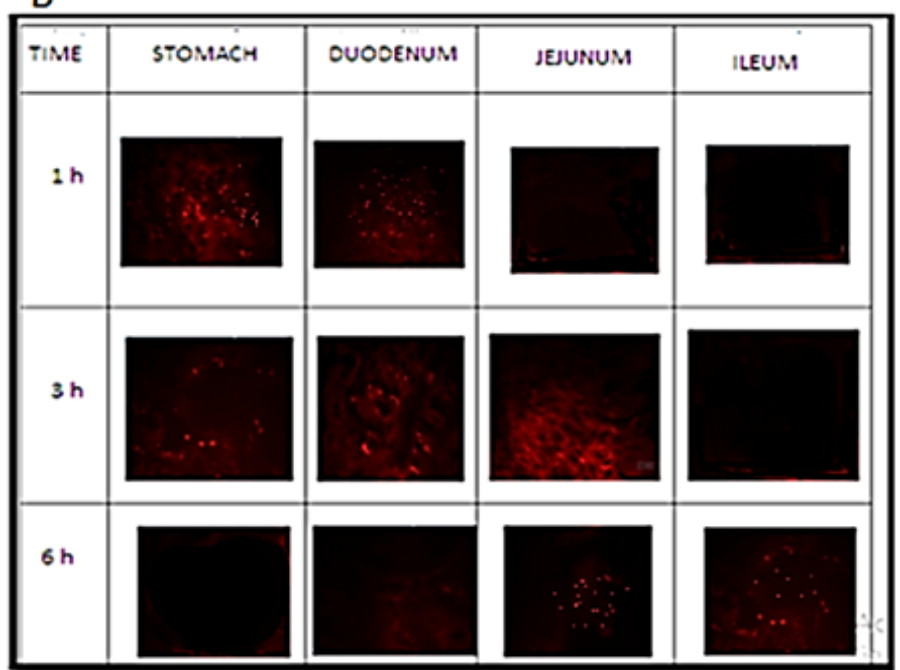

Figure 5: Fluorescence microscopic images from histo-pathological slides of stomach and different parts of the intestine at various time intervals showing gastrointestinal transit of F1 microspheres (A) and F5 microspheres (B).

hence the rate of release was slower. The lower polymer concentration allows easy release of the drug due to the lower diffusional resistance. The release profiles also show that there is a marked increase in drug release from the Polycarbophil microspheres as compared to similar drug to polymer ratios of the carbopol formulations. The drug release from carbopol coated microspheres was slower and more sustained than from polycarbophil coated microspheres in all ratios. This can be attributed to the higher viscosity of the gelled coating of the carbopol 934 microspheres since the polymer is a highly efficient thickener which can retard drug release. The greater cross linking property of carbopol compared to polycarbophil may also be a factor and the principal reason why microspheres of the former polymer have a better surface morphology and spherical shape as compared to the latter. The burst release that is observed from both types of formulations in the first 30 min of the study is probably from the drug-saturated resin surface. This saturation could have occurred in the short interval of time between the drug-resin complexation and the encapsulation of the particles.

Drug release from all the formulations followed first order kinetics. On analyzing the data by the Korsmeyer-Peppas, it is observed that the ' $n$ ' values for all the formulations ranged between $0.4732-0.5028$ which indicate that the mechanism of drug release could be described by nonFickian diffusion. ${ }^{27}$ Drug release from all the formulations can also be described by Higuchi model, since regression values for this model were above 0.95 , hence drug release from these formulations appeared to follow matrix diffusion process.

Fluorescence images of the prepared histo-pathological slides of stomach and intestine showed major distribution of the particles (seen as orange spots in the dark background of the images) in the stomach in the first hour after administration. For both formulations, very few particles were found in the duodenum and none in the jejunum and ileum. After $3 \mathrm{~h}$, more particles were visible in the duodenum, a few in the jejunum and none in the ileum. In case of carbopol particles, after $6 \mathrm{~h}$ more microspheres were found in the duodenum than in the stomach. This is probably because these microspheres had released their resin cores after disintegration of the mucoadhesive coat. Thus they are found mostly in the duodenum and jejunum. A few particles were found in the ileum and some still remained in the stomach. Although the movement of the polycarbophil microspheres was similar to the carbopol microspheres, however the former showed faster movement since the fluorescence images revealed the presence of greater number of particles in the ileum at the end of $6 \mathrm{~h}$. This study confirms the fact that the mucoadhesive microspheres of clarithromycin showed gastro retention for $6 \mathrm{~h}$ in the case of carbopol microspheres and at least $4 \mathrm{~h}$ in the case of polycarbophil microspheres. These results were consistent with the percentage mucoadhesion of $60-68 \%$ achieved in the in vitro wash off test using sheep intestinal mucosa at the end of $6 \mathrm{~h}$ for both formulations. The data from in vitro drug release studies in simulated gastric fluid indicates that F1 and F5 released more than $50 \%$ of the complexed drug in $6 \mathrm{~h}$. Therefore it can be assumed that as much drug would be available to the intestine for absorption during the period of gastro-retention.

\section{CONCLUSION}

Results have shown that formulations with carbopol may be more successful in gastro-retention than those of polycarbophil, while providing sustained release for more than $12 \mathrm{~h}$. The use of ion exchange resins for drug complexation in combination with mucoadhesive coating of the resulting microspheres can be used as a novel technique for the gastro-retentive delivery of ionizable drugs such as clarithromycin. Higher local concentrations of clarithromycin achieved with this mode of delivery can help to effectively target the H. pylori and thereby achieve better control over gastric ulcers or gastritis.

\section{ACKNOWLEDGEMENT}

The authors would like to thank the NITTE University, Mangalore for providing the necessary facilities for carrying out this investigation.

\section{CONFLICT OF INTEREST}

The authors declare that there are no conflicts of interest.

\section{ABBREVIATIONS}

USP: United States Pharmacopoeia; GRDDS: Gastro-Retentive Drug Delivery System; GIT: Gastro Intestinal Tract; SEM: Scanning electron microscopy; IAEC: Institutional Animal Ethics Committee.

\section{REFERENCES}

1. Singh RP, Rathore D. Gastroretention a means to address local targeting in the gastric region. Pharmacophore. 2012;3(6):287-300

2. Kumar A, Alok S, Mourya A, Kumar R, Mishra A. Development and charecterization of gastroretentive multiparticulate delivery system of Amoxiclin-Resinate complex. Int J Pharm Sci Res. 2016;7(2):738-51. 
3. Pant P, Bansal K, Therdana RR, Padhee P. Formulation design and evaluation of gastroretentive mucoadhesive microspheres of clarithromycin. Int J Res Pharm Chem. 2011;1(3):2231-781.

4. Dave BS, Amin AF, Patel MM. Gastro retentive drug delivery system of ranitidine hydrochloride: formulation and in vitro evaluation. AAPS Pharm Sci Tech. 2004;5(2):34.

5. Gaur P, Mishra S, Bhardwaj S, Puri D, Kumar S. Ion Exchange Resins in Gastroretentive Drug Delivery: Characteristics, Selection, Formulation and Applications. J Pharm Sci Pharmacol. 2014;1(4):304-12.

6. Anand V, Kandarapu R, Garg S. lon-exchange resins: Carrying drug delivery forward. Drug Discovery Today. 2001;6(17):905-14.

7. Kumar A, Sing N, Kaushik D. Taste masking of clarithromycin using complexation with lon exchange resin. Int J Pharm Tech Res. 2014;6(1):203-11.

8. O'Connor A, Gisbert J, McNamara D, O'Morain C. Treatment of Helicobacter pylori Infection 2010. Helicobacter. 2010;15:46-52.

9. Mejia A, Kraft W. Acid peptic diseases: Pharmacological approach to treatment. Expert Rev Clin Pharmacol. 2009;2(3):295-314.

10. Lobenberg R, Amidon GL. Modern bioavailability, bioequivalence and biopharmaceutics classification system: New scientific approaches to international regulatory standards. Eur J Pharm Biopharm. 2000;50(1):3-12.

11. Pereira J, Mejia-Ariza R, llevbare G, McGettigan H, Sriranganathan N, Taylor L, et al. Interplay of Degradation, Dissolution and Stabilization of Clarithromycin and Its Amorphous Solid Dispersions. Mol Pharm. 2013;10(12):4640-53.

12. Rajinikanth P, Karunagaran L, Balasubramaniam J, Mishra B. Formulation and Evaluation of Clarithromycin Microspheres for Eradication of Helicobacter pylori. Chem Pharm Bull. 2008;56(12):1658-64.

13. Chaudri PD, Chaudri SP, Lanke SD, Patel N. Formulation and in vitro evaluation of taste masked orodispesable dosage form of Levocetrizine dihydrochloride. Indian J Pham Educ Res. 2007;41(4):319-28.

14. Cuna M, Alonso MJ, Torres D. Preparation and in vivo evaluation of mucoadhesive microparticle containing amoxicillin-resin complexes for drug delivery to gastric mucosa. Eur J Pharm Biopharm. 2001;51(3):199-205.
15. Patel JK, Bodar MS, Amin PMM. Formulation and optimization of mucoadhesive microspheres of metoclopramide. Indian J Pharm Sci. 2004;66(3):300-5

16. El-Hameed MDA, Kellaway IW. Preparation and charecterization of mucoadhesive polymeric microspheres as intra-nasal delivery system. Eur J Pharm Biopharm. 1997;44:53-60.

17. Singh C, Jain K, Kumar C, Agarwal K. Design and in vitro evaluation of mucoadhesive microcapsules of pioglitazone. J Young Pharm. 2009;1(3):195.

18. Dasankoppa FS, Komal S, Sholapur H, Swamy N, Sajjanar V. Design, Optimization and Evaluation of Chewable Tablets of Clarithromycin using lon Exchange Resins. Indian J Pharm Sci. 2016;78(6):818-26.

19. Peppas N, Narasimhan B. Mathematical models in drug delivery: How modeling has shaped the way we design new drug delivery systems. J Control Release. 2014; 190:75-81.

20. Shah S, Pandya S. Molecular Properties Evaluation of Indion 234-Ondansetron Resinates. J Young Pharm. 2010;2(3):247-51.

21. Calvet X. Dealing with uncertainty in the treatment of Helicobacter pylori. Ther Adv Chronic Dis. 2018;9(4):93-102.

22. Nakanishi T, Kaiho F, Hayashi M. Use of sodium salt of carbopol 934P in oral peptide delivery. Int J Pharm. 1998;171(2):177-83.

23. Ch'ng HS, Park H, Kelly P, Robinson JR. Bioadhesive polymers as platforms for oral controlled delivery II: Synthesis and evaluation of some swelling, waterinsoluble bioadhesive polymers. J Pharm Sci. 1985;74(4):399-405.

24. Verma RK, Garg S. Development and evaluation of osmotically controlled oral drug delivery system of Glipizide. Eur J Pharm Biopharm. 2004;57(3):513-25.

25. Bera K, Mazumder B, Khanam J. Study of the Mucoadhesive Potential of Carbopol Polymer in the Preparation of Microbeads Containing the Antidiabetic Drug Glipizide. AAPS Pharm Sci Tech. 2016;17(3):743-56.

26. Park K, Jeong HS. Drug loading and release properties of ion exchange resin complexes as drug delivery matrix. Int J Pharm. 2008;361(1-2):26-32.

27. Peppas NA. Analysis of Fickian and non Fickian drug release from polymers. Pharm Acta Helv. 1985;60(4):110-1

Article History: Submission Date : 03-08-2020; Revised Date : 29-09-2020; Acceptance Date : 11-10-2020.

Cite this article: Stephin J, Marina K. Preparation and Investigation of Gastro-Retentive Mucoadhesive Microspheres of Clarithromycin-Resin Complex. Int. J. Pharm. Investigation, 2020;10(4):445-51. 